(IBD) with a variable clinical course. Common findings include enthesitis and dactylitis. Current treatments include anti-TNFa and anti-IL-12/IL-23 antibodies with varying success rates but the involvement of several pro-inflammatory cytokines suggests that other targeted therapies may be effective. Notably, the JAKs (a family of 4 non-receptor tyrosine kinases) are crucial for the signaling of many pro-inflammatory cytokines. In this regard, the JAK1-selective inhibitor filgotinib (GLPG0634, GS-6034) demonstrated clinical efficacy in patients with rheumatoid arthritis, a disease that shares some hallmarks with PsA and Crohn's disease, making this molecule a potential therapeutic tool for the treatment of PsA.

Objectives: Filgotinib was evaluated at the dose of $30 \mathrm{mg} / \mathrm{kg} / \mathrm{d}$ (per os) in a mouse model of PsA induced by overexpression of IL-23.

Methods: Overexpression of IL-23 was induced by hydrodynamic delivery of mIL23 enhanced Episomal Expression Vector (SBI) to male B10.RIII mice ${ }^{1}$. Evolution of inflammation of the paws and fingers was assessed by clinical scoring as well as in vivo molecular imaging (Bruker In-Vivo Xtreme imaging system). Enthesis and fingers were collected for expression analysis of inflammatory genes and targetrelated biomarkers. Neutrophil infiltrate, as well as pSTAT3 positive cells, were analyzed using immunohistochemistry in Achilles' enthesis and subcutaneous area, respectively. Colon was collected for lesion score determination as well as inflammatory and target-related biomarker gene expression.

Results: High levels of IL-23 were maintained during the time-course of the study and were correlated with severity of finger and paw swelling. Localization of the fluorescent signal using ProSense ${ }^{\mathrm{TM}}$ imaging was asscociated with inflammation of enthesis and finger reported in PsA. Moderate inflammation of the colon was also observed. Filgotinib significantly improved clinical scoring and tended to prevent neutrophil/granulocyte infiltrate in paw (with significant effect being showed at earlier time point). Filgotinib reversed some up-regulated inflammatory genes in enthesis and/or fingers (CCL20, CXCL1, IL-22, MMP9 and TNFa) and reduced the target-related gene Mx2. Filgotinib significantly counteracted PSTAT3 induction in the subcutaneous area further demonstrating target engagement in the diseased tissue. Finally in line with previous findings ${ }^{2}$, Mx2 expression in colon was slightly reversed by filgotinib.

Conclusions: In a mouse model of PsA, filgotinib improved global clinical score and decreased signs of inflammation in hindlimbs. Target engagement both in hindlimbs and colon was also demonstrated. These data support the evaluation of filgotinib in patients with PsA.

References:

[1] Sherlock et al. 2012 Nature Med 7:1069-1076.

[2] Van Rompaey et al. $2013 \mathrm{~J}$ Immunol. 191:3568-3577.

Disclosure of Interest: C. Robin-Jagerschmidt Employee of: Galapagos SASU, S. Lavazais Employee of: Galapagos SASU, F. Marsais Employee of: Galapagos SASU, A. Monjardet Employee of: Galapagos SASU, A. Cauvin Employee of: Galapagos SASU, C. Saccomani Employee of: Galapagos SASU, I. Parent Employee of: Galapagos SASU, D. Merciris Employee of: Galapagos SASU, E. Chanudet Employee of: Galapagos SASU, M. Borgonovi Employee of: Galapagos SASU, L. Lepescheux Employee of: Galapagos SASU, M. Auberval Employee of: Galapagos SASU, S. Dupont Employee of: Galapagos SASU, P. Clement-Lacroix Employee of: Galapagos SASU, R. Galien Employee of: Galapagos SASU DOI: 10.1136/annrheumdis-2017-eular.1977

THURSDAY, 15 JUNE 2017

Bringing rheumatology research to the next level: addressing the main challenges of patient partnerships in research and health care service design

\section{OP0162-PARE AN EXPLORATION OF LIVED EXPERIENCES AMONGST ADULTS WITH RHEUMATOID ARTHRITIS USING AN ONLINE RESEARCH COMMUNITY PLATFORM: A PILOT STUDY}

S.R. Stones ${ }^{1,2,3}$, S. Bull ${ }^{1}$, S. Becerra ${ }^{1} .{ }^{1}$ Double Helix Consulting, Macclesfield; $\frac{1}{2}$ Faculty of Life Sciences, The University of Manchester, Manchester; ${ }^{3}$ School of Healthcare, University of Leeds, Leeds, United Kingdom

Background: Online technology has revolutionised the way in which people connect and share their experiences. It also brings new opportunities to engage patients in health and social care research through the use of an online research community platform (ORCP). For example, it can improve the accuracy and usefulness of information gathered about research priorities, and it can be used to understand behaviours and preferences. Given an increasing prevalence of long-term conditions including rheumatoid arthritis, online technology represents a novel route for participation and engagement in research.

Objectives: To explore the benefits and limitations of an ORCP through understanding lived experiences of adults with rheumatoid arthritis.

Methods: We used a purposive sampling approach to ensure variation of key attributes amongst people with rheumatoid arthritis. A total of eight individuals used the ORCP during the pilot study. Qualitative data were collected through online focus groups, conducted as semi-structured interviews and asynchronous threaded discussions. The study was conducted in line with fieldwork guidelines, and written informed consent was obtained.

Results: The closed ORCP enabled a physically disconnected group to share their experiences of living with rheumatoid arthritis, describing the symptoms, diagnostic experience and support they received. In addition, participants shared their experiences and opinions about treatment delivery and adherence, the impact of rheumatoid arthritis, and the experiences of transitional care from paediatric to adult health services, where appropriate. Reasons and feeling about research participants and drug development processes were also discussed.

Conclusions: Our pilot study provided important accounts from people living with rheumatoid arthritis, highlighting the substantial impact of the disease on their daily lives. The ORCP removed physical contact between the researcher and participants, the absence of which may enable a richer data collection. However, it also has its limitations, primarily because the researcher is less able to gauge participants' attitudes and concerns. ORCPs represent a novel route of data collection, enabling researchers to immerse themselves into a community of individuals, whether they be patients, carers or professionals.

Acknowledgements: The authors would like to thank the individuals who contributed their thoughts and experiences to the pilot study.

Disclosure of Interest: S. Stones Employee of: McCann Complete Medical., S. Bull Employee of: McCann Complete Medical., S. Becerra Employee of: McCann Complete Medical.

DOI: 10.1136/annrheumdis-2017-eular.1029

\section{OP0163-PARE INVIGORATING THE PRINCIPLE OF PARTICIPATORY RESEARCH IN GERMANY - SETUP OF A TRAINING COURSE FOR PATIENT REPRESENTATIVES}

J. Clausen. Deutsche Rheuma-Liga Bundesverband, Bonn, Germany

Background: Patient participation in research projects is important because it enhances the legitimacy of research projects and facilitates the implementation of research results after completion of the respective projects. Since 2009, patient representatives have been actively involved in EULAR projects, and the first EULAR training course for patient representatives was conducted in 2010.

Objectives: The aim of the project was to create a training course for Germanspeaking patient representatives and thereby to invigorate the principle of participatory research in Germany. The training course is supposed to enable patients to make valuable contributions in research projects. In addition the training course aimed at lowering barriers and strengthening the patients' selfconfidence, in order to facilitate their integration in the unfamiliar environment among researchers.

Methods: Participants were trained during a two-day interactive training course. For evaluation of the course, each participant anonymously answered 14 questions in a questionnaire.

Results: The training course consists of seven modules. In the first module (I), the history of the EULAR "patient research partners" is described and the tasks of the future German patient research partners are outlined. In the following modules, various types of research and study designs (II), the generation of research questions and hypotheses (III), various scientific tools (IV), the detailed sequence of steps in a typical research project $(\mathrm{V})$, literature research in scientific databases $(\mathrm{VI})$ and the process of reviewing grant applications (VII) are explained. Each module is subdivided in an explanatory section, an exercise section (where the participants have to apply the newly achieved skills) and a final discussion section.

So far, two courses have been conducted. The training course was rated either "very good" or "good" by $77 \%$ and $23 \%$ of the participants, respectively. Those patients, already actively involved in research projects, acclaim participatory research as interesting and enriching

Conclusions: The training course was perceived very well by the participants. In future follow-up meetings, the usefulness of the various modules and any missing items will be discussed and the training course adapted accordingly.

Acknowledgements: We thank Dr. C. Sander for her contribution to the training course.

Disclosure of Interest: None declared

DOI: 10.1136/annrheumdis-2017-eular.1923

\section{THURSDAY, 15 JUNE 2017}

\section{Osteoarthritis: new horizons for treatment}

\section{OP0164 OPTIMIZING RECRUITMENT CRITERIA FOR AN OSTEOARTHRITIS STRUCTURE MODIFICATION TRIAL: DATA FROM THE OAI}

M.A. Bowes ${ }^{1}$, G. Guillard ${ }^{1}$, A. Brett ${ }^{1}$, G.R. Vincent ${ }^{1}$, P.G. Conaghan ${ }^{2}$ ${ }^{1}$ Imorphics, Manchester; ${ }^{2}$ Leeds Institute of Rheumatic and Musculoskeletal Medicine, University of Leeds, Leeds, United Kingdom

Background: The design of clinical trials for osteoarthritis is challenging; structural changes in tissues are quantitatively small and proceed very slowly. No clear guidance exists on how to optimise recruitment. $K L$ grade is a poor recruitment criterion as centres interpret KL differently. Quantitative measures should be 
better, and metric radiographic joint space width (rJSW) is related to subsequent risk of radiographic progression. Although new MRI measures provide increased responsiveness in DMOAD trials, it is unknown whether selecting for recruitment based on radiographic criteria are well suited for responsiveness of these new measures.

Objectives: (1) To determine which baseline rJSW values are associated with most subsequent progression for rJSW, MRI cartilage and bone outcomes (2) Explore baseline covariates that influence progression rates (3) Estimate the trial numbers needed using the criteria determined by steps (1) and (2).

Methods: We used all knees from the Osteoarthritis Initiative which had all 3 measures recorded (rJSW - Duryea method; MRI cartilage thickness \& bone area, Imorphics) at baseline, 1 and 2 years. We categorised knees into bins of $1 \mathrm{~mm}$ rJSW, and assessed the 2 year changes of each bin, and characterised the distribution of rJSW in KL 0 knees. We used ANCOVA models to consider which covariates (including gender, height, weight, alignment, age, pain severity) affected 2-year slope of change, and responsiveness using SRM. For the final optimised recruitment groups, we calculated SRMs (Cls assessed using the bootstrap method of Efron) and derived the number of patients per arm in a putative trial.

Results: 4796 knees were included (2789 females, mean age 61.45). The lower 95th percentile values for rJSW in women and men were 3.9 and $4.5 \mathrm{~mm}$ respectively. The mean changes at 2-years for all 3 outcomes were greatest for the categories of $2-3$ and $3-4 \mathrm{~mm}$ baseline rJSW (Figure $1 \mathrm{~A}$ ) with notably little change in knees with rJSW $<2 \mathrm{~mm}$. Of the covariates, only pain improved responsiveness. Using a total WOMAC pain criterion; $\geq 3 / 20$ reduced numbers from 726 knees to 331 knees, and improved 1 year SRM $(95 \% \mathrm{Cl}$ ) from 0.27 $(0.17,0.34)$ to $0.41(0.29,0.51)$ in rJSW, from $0.45(0.37,0.51)$ to $0.55(0.45,0.65)$ in MRI cartilage and from $0.60(0.52,0.66)$ to $0.73(0.62,0.83)$ in MRI bone. Figure 1B shows the relative SRMs for the 3 outcomes based on 2 inclusion criteria (rJSW $2-4 m m$ and pain $>3 / 20 ; n=331$ ) and demonstrates the required trial numbers (with confidence intervals) based on the SRMs.
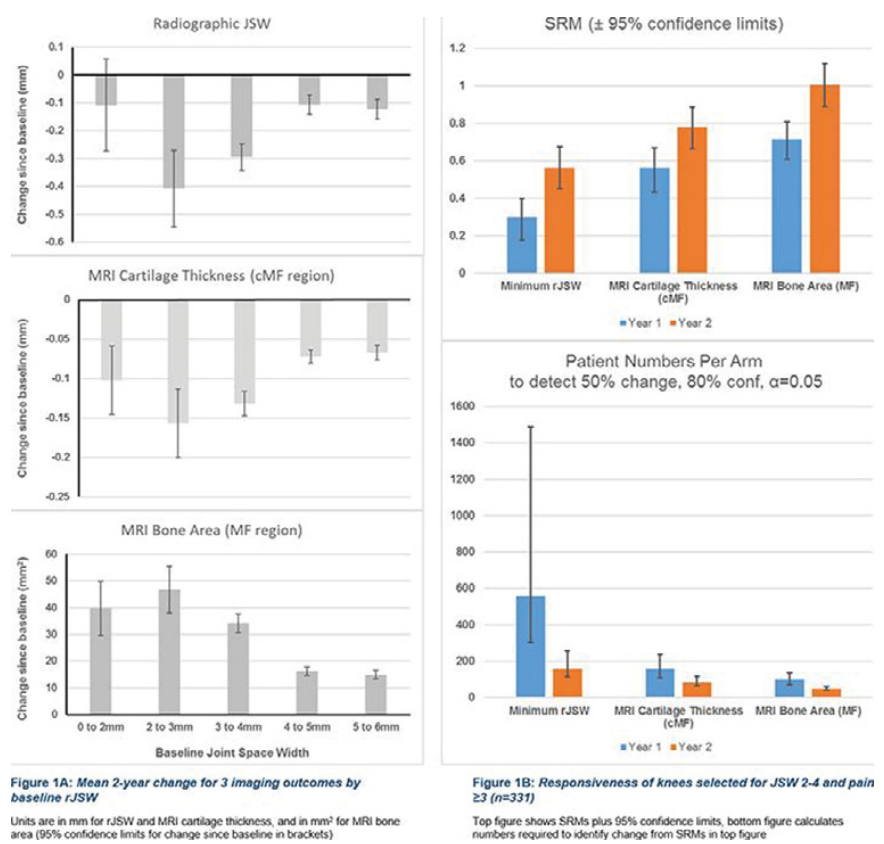

Conclusions: Selecting patients based on 2 simple criteria will improve responsiveness in clinical trials for all 3 imaging outcomes using standard imaging outcomes. Selecting for rJSW of $2-4 \mathrm{~mm}$ is most important while adding a pain criteria further improves responsiveness; no other covariates improved this. Caution should be applied when using SRM to power a study because of the inherent difficulties in calculating standard deviations; Fig 1B shows for example that the confidence limits for rJSW at 12 months vary from 302 to 1489 . This analysis also confirms the advantages of MRI outcomes over rJSW in terms of study size and duration: a 12-month study with cartilage thickness or bone area endpoints needs no more than 238 or 137 patients (including the upper 95th percentile confidence limit).

Disclosure of Interest: M. Bowes Employee of: Imorphics Ltd, G. Guillard Employee of: Imorphics Ltd, A. Brett Employee of: Imorphics Ltd, G. Vincent Employee of: Imorphics Ltd, P. Conaghan: None declared

DOI: 10.1136/annrheumdis-2017-eular.6860

\section{OP0165 A NEOEPITOPE FRAGMENT OF C-REACTIVE PROTEIN IS PROGNOSTIC OF RADIOGRAPHIC KNEE OA}

A.C. Bay-Jensen ${ }^{1}$, A. Bihlet ${ }^{2}$, I. Byrjalsen ${ }^{2}$, J.R. Andersen ${ }^{2}, \mathrm{Y}^{2} \mathrm{He}^{1}$, A.S. Siebuhr ${ }^{1}$, C.S. Thudium ${ }^{1}$, B.J. Riis ${ }^{2}$, C.H. Christiansen ${ }^{2}$, M.A. Karsdal ${ }^{1}$. ${ }^{1}$ Rheumatology, Nordic Bioscience Biomarkers and Research; ${ }^{2}$ Clinical development, Nordic Bioscience, Herlev, Denmark

Background: There are two major needs in clinical development of DMOADs: 1) Identifying a suitable population with an active and progressive disease in order to demonstrate significant improvements by an efficacious intervention; and 2) Phenotyping patients and linking them to a corresponding treatment mode-ofaction (e.g. anti-inflammatory). The biochemical marker CRPM is a neoepitope of $\mathrm{C}$-reactive protein (CRP) and is released from the local inflamed tissue when CRP is degraded by proteases such as matrix metalloproteinases

Objectives: The purpose of this study was to translate the CRPM, from rheumatoid arthritis (RA), where it has been extensively tested, to OA, and to test whether it is predictive of radiographic $O A$

Methods: The placebo arms of two phase III OA trial (clinicaltrial.gov: NCT00486434 and NCT00704847), a phase III RA study (the LITHE study, $\mathrm{N}=490$ ), which included patients with active, moderate-severe RA (NCT00106535), as well as in an early $\mathrm{RA}$ cohort $(\mathrm{N}=92)$ were used. Subjects with symptomatic and radiographic knee OA: WOMAC pain $\geq 150 \mathrm{~mm}$ and/or WOMAC function $\geq 510 \mathrm{~mm}$, and Kellgren-Lawrence grade $(\mathrm{KLG}) 2$ or 3 . KLG were scored for both knees at baseline and year 2 (Y2). Serum CRPM and hsCRP were measured at baseline. The association between serum CRPM levels and disease activity score (DAS28) and hsCRP was investigated by Spearman's correlations. Quartile ranges of CRPM in the early RA cohort were used to define the cut-off between inflammatory $\mathrm{OA}$ and non-inflammatory $\mathrm{OA}$. OA knees were divided into cases and controls based on a terminology proposed by the FNIH-OAI consortium (1); knees with $K L G \geq 2$ at $B L$ were excluded, and incidence $O A$ at $Y 2$ was defined $K L G \geq 2$. Logistic regression was used to compare cases and controls.

Results: There was a significant correlation between disease activity measures and CRPM in both RA studies. Seventy-five percent of the LITHE patients had high or very high levels of CRPM at BL, which was changed to a pattern similar to early RA after treatment. The mean CRPM levels were significantly lower in $\mathrm{OA}(8.5[95 \% \mathrm{Cl} 8.3-8.8] \mathrm{ng} / \mathrm{mL})$ compared to the RA patients (15.6 [9.5-21.6] $\mathrm{ng} / \mathrm{mL})$; however, a significant subset of OA patients (41\% and $31 \%$ in SMC2301 and SMC2302) had CRPM levels $>9 \mathrm{ng} / \mathrm{mL}$, as $75 \%$ of patients with early RA. Patients with $\mathrm{BL}$ or $\mathrm{Y} 2 \mathrm{CRPM}$ levels $\geq 9 \mathrm{ng} / \mathrm{mL}$ were more likely to develop knee $\mathrm{OA}$ than patients with low level of CRPM. Overall, moderate to very high levels of CRPM at BL and Y2 were predictive of incidence OA with odds ratio of 4.6 [1.2-17] and 2.5 [1.2-4.8].

\begin{tabular}{|c|c|c|c|c|c|c|}
\hline \multicolumn{2}{|l|}{ Table } & \multirow{2}{*}{$\begin{array}{l}\text { Early RA } \\
0.54(<0.0001)\end{array}$} & \multicolumn{2}{|c|}{ Moderate-Severe RA } & \multirow[t]{2}{*}{ SMC2301 } & \multirow[t]{2}{*}{ SMC2302 } \\
\hline hsCRP & Rho (p-value) & & $0.66(<0.0001)$ & & & \\
\hline DAS28 & Rho (p-value) & $0.43(<0.0001)$ & $0.24(<0.0001)$ & & & \\
\hline \multirow{2}{*}{\multicolumn{2}{|c|}{ CRPM level }} & Quartile range & \multicolumn{4}{|c|}{ No. of patients (\%) } \\
\hline & & & Before treatment & After treatment & $n_{1}$ & $n_{2}$ \\
\hline \multicolumn{2}{|l|}{ Low } & $<9 \mathrm{ng} / \mathrm{mL}$ & $49(8.2 \%)$ & $87(18 \%)$ & $267(59 \%)$ & $233(69 \%)$ \\
\hline \multicolumn{2}{|c|}{ Moderate } & $9-12$ & $100(17 \%)$ & $123(25 \%)$ & $137(31 \%)$ & $71(21 \%)$ \\
\hline \multicolumn{2}{|l|}{ High } & $12-15$ & $132(22 \%)$ & $105(21 \%)$ & $26(6 \%)$ & $23(7 \%)$ \\
\hline \multicolumn{2}{|c|}{ Very high } & $>=15$ & $318(53 \%)$ & $175(36 \%)$ & $19(4 \%)$ & $11(3 \%)$ \\
\hline
\end{tabular}

Conclusions: A subset of OA patients, up to $41 \%$, appear to have tissue inflammation comparable to that of RA, reflected by the level of CRPM. Furthermore, high CRPM levels was prognostic of incident knee OA. These data suggest that CRPM is blood-based biochemical marker for finding OA patients with an inflammatory phenotype.

References:

[1] Longitudinal validation of periarticular bone area and 3D shape as biomarkers for knee OA progression? Data from the FNIH OA Biomarkers Consortium.Hunter D et al.Ann Rheum Dis. 2016 Sep;75(9):1607-14.

Disclosure of Interest: A. Bay-Jensen Shareholder of: Nordic Bioscience, Grant/research support from: DBOARD, Employee of: Nordic Bioscience, A. Bihlet Shareholder of: Nordic Bioscience, Employee of: Nordic Bioscience, I. Byrjalsen Employee of: Nordic Bioscience, J. Andersen Shareholder of: Nordic Bioscience, Employee of: Nordic Bioscience, Y. He Employee of: Nordic Bioscience, A. S. Siebuhr Employee of: Nordic Bioscience, C. Thudium Employee of: Nordic Bioscience, B. Riis Shareholder of: Nordic Bioscience, C. Christiansen Shareholder of: Nordic Bioscience, M. Karsdal Shareholder of: Nordic Bioscience, Employee of: Nordic Bioscience

DOI: 10.1136/annrheumdis-2017-eular.6290 